\title{
e-Phaïstos
}

e-Phaïstos

Revue d'histoire des techniques / Journal of the history

of technology

I-1 | 2012

Le patrimoine maritime

\section{Signatures architecturales vs. Spécificités régionales au sein de la tradition de construction navale ibéro-atlantique}

\section{Vanessa Loureiro}

\section{OpenEdition}

\section{Journals}

Édition électronique

URL : http://journals.openedition.org/ephaistos/234

DOI : 10.4000/ephaistos.234

ISSN : 2552-0741

\section{Éditeur}

IHMC - Institut d'histoire moderne et contemporaine (UMR 8066)

\section{Édition imprimée}

Date de publication : 1 juin 2012

Pagination : 27-38

ISSN : 2262-7340

\section{Référence électronique}

Vanessa Loureiro, «Signatures architecturales vs. Spécificités régionales au sein de la tradition de construction navale ibéro-atlantique », e-Phaïstos [En ligne], I-1 | 2012, mis en ligne le 01 janvier 2015, consulté le 24 avril 2019. URL : http://journals.openedition.org/ephaistos/234 ; DOI : 10.4000/ ephaistos.234 


\title{
Signatures architecturales vs. Spécificités régionales au sein de la tradition de construction navale ibéro-atlantique
}

\author{
Vanessa Loureiro \\ Instituto de Arqueologia e Paleociências das \\ Universidades Nova de Lisboa e do Algarve
}

\section{Introduction}

La tradition ibéro-atlantique traduit la pratique de construction de navires à bordé à franc-bord sur squelette, diffusée dans les espaces maritimes européens de la Renaissance sous la dénomination de construction "à carvel”, "à la manière de la caravelle".

À la base du concept de tradition de construction navale, il n'existe rien de plus qu'un schéma de classification qui cherche à réduire la réalité multidimensionnelle à un modèle généraliste. Il faut néanmoins être attentif à la complexité des schémas de classification. D'une part, l'extrême simplification ne permet de tirer aucune conclusion, puisque n'importe quelle caractéristique formelle, à moins d'être définie avec précision, peut être détectée dans diverses pratiques de construction navale. D'autre part, chaque embarcation est, d'une certaine façon, unique et peut, en dernier ressort, constituer une catégorie isolée.

Sous-jacente au concept de tradition de construction navale est, en fait, la notion de groupe polythétique $^{1}$, la similarité entre deux entités ou individus quelconques se fondant sur un nombre élevé de variables, même si aucun attribut n'est partagé par tous les membres. Ce qui signifie que, dans le cadre de toute tradition de construction navale, certaines embarcations pourront présenter toutes les signatures architecturales requises, alors que d'autres seront dépourvues d'une ou de deux caractéristiques, ou même qu'elles présenteront des preuves peu claires. Toutefois, c'est la combinaison générique et non pas individuelle des signatures architecturales qui autorise l'affiliation d'un ensemble d'embarcations à une tradition de construction navale.

Dans l'identification des signatures architecturales d'une tradition de construction navale, la distinction entre principes et processus de construction revêt donc une importance particulière. Les principes de construction traduisent le projet et l'intention du constructeur (le système architectural de l'embarcation), alors que les processus de construction interprètent la pratique de chantier, les méthodes de construction et les solutions adoptées pour résoudre les divers problèmes auxquels le constructeur s'est trouvé confronté ${ }^{2}$.

En effet, les différences technologiques observées parmi les embarcations affiliées à une même tradition de construction navale sont significatives. Cette 
variabilité peut être due au paysage culturel maritime des navires proprement dits (différentes fonctions et/ou environnements opérationnels), à des spécificités à caractère régional (différents niveaux d'accès aux matières premières et aux produits annexes et/ou de capacités de transfert technologique) ou encore à des processus évolutifs qui ont été enregistrés progressivement au cours du temps. Indépendamment de leurs variations technologiques, ces embarcations présentent néanmoins un système architectural similaire.

\section{Signatures architecturales de la tradition de construction navale ibéro-atlantique}

Le concept de tradition de construction navale ibérique n'a pas plus de deux décennies. Il a été développé par Thomas Oertling afin de caractériser une classe de navires, y compris des caravelles, des nefs et des galions, qui avaient en commun des méthodes et des processus de construction ainsi qu'une intention présidant à leur conception, la navigation océanique ${ }^{3}$.

À partir de l'analyse des principales caractéristiques de construction de sept épaves archéologiques - Rye A, San Juan, Cattewater, San Esteban, Highborn Cay, Molasses Reef et Studland Bay - Oertling a développé une matrice théorique et comparative pour cette tradition de construction qu'il a désignée par Atlantic Vessel. La modification de la désignation Atlantic Vessel en tradition ibéro-atlantique, proposée par Francisco Alves et Éric Rieth au cours du Symposium International de Lisbonne de 1998, n'a pas impliqué de changement de paradigme ${ }^{4}$, bien qu'il ait été attribué clairement à cette tradition de construction navale une origine méditerranéenne et médiévale et qu'un postulat ait été émis, le développement, dans les zones atlantiques de la péninsule Ibérique, de détails techniques sui generis leur conférant une identité effective ${ }^{5}$.

Fondée sur la systématisation d'un ensemble de caractéristiques de construction plus ou moins communes aux sept épaves archéologiques analysées, la perspective théorico-comparative d'Oertling représentait, à la lumière de l'état de la recherche sur l'architecture navale post-médiévale et moderne dans les années
1980, une avancée des connaissances sur les navires responsables de l'expansion maritime des royaumes ibériques aux $\mathrm{XV}^{\mathrm{e}}$ et $\mathrm{XVI}^{\mathrm{e}}$ siècles. La matrice proposée était composée au départ de 12 signatures :

-les varangues centrales fixées aux premiers genoux, avant leur mise en place sur la quille, à l'aide de tenons en queue d'aronde, de chevilles en bois et de clous ;

-le bordé fixé à la charpente transversale à l'aide d'une combinaison régulière de clous en fer et de chevilles en bois ;

-le talon en tant qu'élément de liaison de la quille à l'étambot ;

-la courbe d'étambot en tant qu'élément de renfort des liaisons de la quille et de l'étambot au talon ;

-les fourcats positionnés sur la courbe d'étambot à l'aide de lisses ;

-la carlingue encastrée sur la face supérieure des varangues à l'aide d'encoches ouvertes dans sa surface inférieure ;

-l'emplanture du mât intégrée dans la carlingue ; -l'emplanture du mât entourée sur les côtés par des taquets et des serres ;

-le vaigrage couvrant la surface des varangues ; -des moques en forme de larme supportant les haubans ;

-le tableau arrière plat parachevant l'étambot ; -les galbords taillés dans des planches en bois plus épaisses que le reste du bordé.

La seule modification dans cette perspective a été sa réduction à onze signatures architecturales, la dernière ayant été éliminée ${ }^{6}$. Mais, en revanche, chacune des caractéristiques énoncées a été peu à peu élargie afin d'intégrer, dans une même signature, des variations qui avaient été identifiées dans les épaves ajoutées à la matrice?.

L'élargissement du spectre de la perspective théorico-comparative d'Oertling a permis effectivement d'englober une partie de la complexité de la tradition ibéro-atlantique. Toutefois, la dernière matrice publiée par cet auteur ${ }^{8}$, et utilisée successivement comme 
référence par des chercheurs dans le monde entier, présente aujourd'hui des inconstances en ce qui concerne la caractérisation de certains des sites archéologiques et est loin de représenter la totalité des navires ibéroatlantiques connus9.

La perspective théorico-comparative que nous proposons maintenant résulte non seulement de l'observation directe des processus et des méthodes de construction sous-jacentes aux 23 épaves ibéro-atlantiques identifiées dans le monde ${ }^{10}$, mais encore du relevé des divers systèmes architecturaux et de leur comparaison avec les sources écrites des $\mathrm{XVI}^{\mathrm{e}}$ et XVII ${ }^{\mathrm{e}}$ siècles.

L'analyse des traités de construction navale révèle comme caractéristique distinctive de cette tradition le recours à des processus géométriques pour définir les principales lignes longitudinales et transversales des navires. Bien qu'au moins jusqu'à la moitié du XVII siècle il n'existait pas de plans ou dessins de navires, on ne peut ignorer l'existence d'un modèle mental géométrique $^{11}$, probablement diffusé oralement, dont les principales preuves se manifestent dans le recours à un jeu proportionnel entre les dimensions clefs des embarcations et dans l'évolution exponentielle des varangues centrales par rapport à la maîtresse section.

De même, d'autres solutions, comme l'utilisation de lisses, le fait de tirer parti de la tension naturelle du bois pour fixer des éléments structurels et le recours à un tableau arrière plat révèlent une «proto-industrialisation ${ }^{12}$ du processus de construction qui doit être entendue comme une caractéristique essentielle du système architectural ibéro-atlantique.

Prétendant procéder à une révision scientifique de la matrice d'Oertling, nous proposons ci-après 14 signatures architecturales pour la tradition ibéro-atlantique :

un jeu proportionnel prédéfini entre les dimensions clefs du navire : creux, fort, longueur hors tout et quille

La littérature des $\mathrm{XVI}^{\mathrm{e}}$ et XVII ${ }^{\mathrm{e}}$ siècles soutient que la conception d'un navire commençait par la définition de la longueur de la quille, à partir de laquelle dérivaient les autres dimensions clefs, longitudinale et transversale, de la structure. Bien que seuls six sites archéologiques (Angra $\mathrm{D}^{13}$, San Juan ${ }^{14}$, Arade $1^{15}$, Emanuel Point ${ }^{16}$, San Diego ${ }^{17}$ et Nossa Senhora dos Mártires ${ }^{18}$ ) aient permis cette analyse métrologique, il a été effectivement observé une constance dans les principaux ratios dimensionnels.

la charpente «un plein, un vide»

La maille serrée de la charpente transversale, sans espacement entre les couples, est une caractéristique clairement attestée dans plus de $60 \%$ des sites archéologiques identifiés, elle confère une robustesse architecturale à la tradition ibéroatlantique. De cette caractéristique découle directement l'importance des proportions du couple (l'épaisseur de l'ensemble varangue et premiers genoux), laquelle est attestée dans les documents contemporains $^{19}$.

la région médiane de la charpente transversale conçue en recourant à des diagrammes prédéfinis

L'existence de varangues prédéterminées ou gabariées est attestée aussi bien dans les sources écrites que dans les sources archéologiques. À partir du dessin de la maîtresse varangue, utilisé comme référence, la diminution de la longueur du plat et l'acculement des varangues prédéterminées, comprises entre la maîtresse section et les varangues du couple de balancement, étaient calculés à l'aide d'échelles exponentielles de valeurs ou gabarits ${ }^{20}$.

les couples centraux montés avant leur mise en place sur la quille

Des observations archéologiques attestent que les couples de la région centrale de l'embarcation sont mis en place sur la quille après le montage des premiers genoux aux varangues, sur la face latérale opposée à la maîtresse section. D'après les sources écrites, seule la maîtresse section possède deux premiers genoux. 
les varangues des extrémités du navire définies à l'aide de lisses

Il résulte de la deuxième caractéristique énoncée que les extrémités de l'embarcation sont définies à l'aide de lisses. Les traités contemporains insistent sur les avantages de l'utilisation de lisses pour accélérer le processus de construction et, dans divers sites archéologiques (e.g. l'Arade $1^{21}$ et le San Juan $^{22}$ ) des marques de clous qui n'apparaissaient pas sur la coque ont été identifiées sur la charpente transversale, l'étrave et l'étambot. La présence de varangues flottantes, non fixées à la quille, témoigne également de l'utilisation de lisses.

le bordé, à franc-bord, installé après la mise en place des couples centraux

Toutes les épaves ibéro-atlantiques dont la structure a pu être analysée attestent la mise en place des couples de la région centrale de l'embarcation avant celui du montage de la coque, ces éléments jouant un rôle déterminant dans la définition de la volumétrie de la carène, alors que le bordé avait un rôle éminemment passif. Par contre, la mise en place des varangues et des premiers genoux des extrémités de poupe et de proue sur la quille ainsi que le montage des deuxièmes genoux et des allonges alternait avec le montage du bordé.

Le bordé à franc bord, calfaté, dont les planches étaient juxtaposées bout à bout, sans présenter le moindre système d'assemblage entre elles, et fixées à la charpente par une combinaison de chevilles en bois et de clous en fer, constitue également l'une des caractéristiques ibéro-atlantiques.

la carlingue, les serres et les préceintes comme éléments de renfort longitudinal

Bien que la charpente transversale joue un rôle actif dans la structure, un ensemble d'éléments longitudinaux garantissait la cohésion du navire, se démarquant de pièces homologues par leur robustesse. Nous nous référons à la carlingue, aux serres et aux préceintes dont l'importance structurelle varie d'un ibéro-atlantique à l'autre, probablement en raison de leur fonction et/ou de leur environnement opérationnel.

des écarts et des tenons comme système de fixation de certains éléments structurels ou de tronçons d'une même pièce

Bien que les traités de construction navale, sauf l'ouvrage de Lavanha, omettent bien souvent le système de fixation des éléments structurels, les navires ibéro-atlantiques se distinguent par le recours intensif aux écarts et aux tenons.

L'utilisation de tenons pour la fixation des genoux aux varangues était déjà considérée par Oertling comme une caractéristique architecturale de cette tradition, en raison de son caractère novateur. Toutefois, il nous semble réducteur de ne pas intégrer cette solution dans un système technique plus vaste, fondé sur des assemblages destinés à assurer la liaison et la transmission d'efforts entre les éléments structurels. La spécificité de la tradition ibéro-atlantique réside dans le fait de tirer parti de la tension du bois pour fixer différentes pièces, ce qui permet d'utiliser des éléments structurels moins longs (en conformité avec la pénurie de bois évoquée dans la péninsule Ibérique du $\mathrm{XVI}^{\mathrm{e}}$ siècle) et de réduire l'investissement dans l'acquisition et/ou la fabrication et le remplacement de chevilles en bois et clous en fer.

le talon en tant qu'élément de liaison de la quille à l'étambot

Dans la plupart des sites ibéro-atlantiques, une profusion de pièces composites a été attestée. Les documents de la Renaissance préconisent de tailler les principaux éléments longitudinaux sous forme de pièces composites de longueur significative, afin de faciliter d'éventuelles réparations.

L'existence d'un élément de liaison entre la quille et l'étambot découle directement de cette recommandation. Néanmoins, le talon revêtait également de l'importance du point de vue des caractéristiques hydrodynamiques.

le vaigrage recouvrant la superfície des varangues

Un pourcentage significatif des épaves ibéro- 
atlantiques analysées présente un vaigrage recouvrant la face supérieure des varangues, qui formait, au niveau de la cale, une plate-forme permettant de ranger la cargaison. La dernière planche du vaigrage, la serre, placée au niveau des extrémités de la varangue, possédait une arête dentée pour encastrer les cloisons, destinées à fermer l'espace entre les premiers genoux et empêcher l'infiltration de détritus dans la charpente. Aussi des planches d'anguiller, planches amovibles, plus étroites que les autres, disposées parallèlement et adossées à la carlingue, permettaient d'inspecter et de nettoyer l'espace entre les membrures.

la carlingue emboîtée sur la face supérieure des varangues par des entailles creusées dans sa surface inférieure

La carlingue dentelée emboîtée, sur l'axe de la quille, dans la région axiale des varangues est l'une des caractéristiques les plus significatives de la tradition ibéro-atlantique, déjà identifiée par Oertling. Nous constatons le parti tiré de la tension naturelle du bois en vue de la cohésion de la structure, motif pour lequel les entailles étaient creusées en fonction de l'épaisseur de chaque varangue et non pas d'après une mesure standard (ce qui aurait facilité le travail de taille).

l'emplanture du mât principal intégrée dans la carlingue et le puisard destiné à recevoir le tube de la pompe taillé, en tout ou partie, dans cet élément

L'une des caractéristiques des navires ibéroatlantiques est l'emplanture du mât principal formée dans une section élargie de la carlingue, placée dans la région médiane de l'embarcation.

Cette caractéristique avait déjà été annoncée par Oertling, y compris le puisard de la pompe taillé partiellement dans l'emplanture du mât. L'analyse des épaves ibéro-atlantiques a toutefois révélé que l'emplacement du puisard varie d'un navire à l'autre, car il est taillé en tout ou partie soit dans l'emplanture du mât (e.g. San Juan et Emanuel Point, respectivement), soit dans la carlingue (e.g.
Rye A).

Il nous semble que l'emplacement du puisard à proximité de l'axe longitudinal et de l'axe transversal de l'embarcation constitue une caractéristique ibéro-atlantique qui reflète les conditions hydrodynamiques et de navigation de ces navires.

l'emplanture du mât entourée sur les côtés par des taquets

Bien que les taquets de l'emplanture du mât ne soient pas attestés dans toutes les épaves ibéroatlantiques, leur récurrence, surtout dans les embarcations de grande taille, est en fait un élément distinctif. Ainsi qu'on le constate sur le plan archéologique, les taquets peuvent prendre la forme d'éléments en coin, emboîtés entre l'emplanture du mât et la serre, ou d'une autre planche du vaigrage ou, encore, ils peuvent être intégrés dans les varangues.

\section{le tableau arrière plat parachevant l'étambot}

L'une des caractéristiques les plus marquées de la tradition ibéro-atlantique est le tableau arrière, plat, en coin par rapport aux flancs de la muraille, qui remplace peu à peu la poupe arrondie. Le tableau recevait le gouvernail axial, caractéristique des navires post-médiévaux et modernes.

L'appréciation des 14 caractéristiques énoncées révèle que la distinction entre les principes et les processus de construction n'est pas triviale. Une caractéristique associée exclusivement, dans le cadre d'une tradition, aux méthodes de construction peut, dans une autre tradition, s'immiscer de telle manière dans son essence qu'elle doive être interprétée comme faisant partie intégrante du système architectural. Ce qui distingue une signature architecturale d'une simple solution de construction est sa récurrence dans une tradition, indépendamment de l'espace et de la chronologie des navires.

Par comparaison avec la matrice d'Oertling, les caractéristiques formulées ici ne représentent pas une inversion du paradigme ibéro-atlantique. Presque tous 
les paramètres définis par cet auteur sont inclus dans la nouvelle formulation, bien qu'ils ne constituent pas nécessairement une preuve architecturale et qu'ils puissent simplement en être le reflet. De même, la nouvelle perspective a une portée supérieure à la matrice d'origine, car elle permet d'intégrer la plus grande partie de la variation architecturale enregistrée dans les épaves.

Il importe en outre encore de mentionner que les caractéristiques énoncées attestent clairement la symbiose entre la construction navale méditerranéenne, solide et enracinée sur toute la façade sud et ouest de l'Europe, et les solutions septentrionales plus appropriées à la navigation océanique et à l'irrégularité du climat des mers du Nord.

En fait, cette tradition n'est pas spécialement novatrice, sa créativité réside davantage sur sa capacité à récupérer des attributs d'autres traditions et à systématiser leur utilisation, afin d'uniformiser et de faciliter les processus de conception et de construction. Aussi les spécificités propres à la tradition ibéro-atlantique semblent être associées à un raisonnement technique et économique, visant à faciliter le processus de construction du navire, à assurer et à augmenter la durabilité de la structure et à en faciliter la réparation.

\section{Spécificités régionales au sein de la tradition ibéro-atlantique}

Vers la fin du XVIe siècle, tout l'espace européen recourait à des embarcations semblables, d'influence ibéro-atlantique, les différences architecturales et de construction attestées relevant de conditions locales de la côte et des ports, du savoir-faire du maitre constructeur et, dans une certaine mesure, des normes techniques liées à la tradition.

Fort négligées jusqu'ici, les variations (les espèces d'arbres, la forme de la quille, les écarts, la typologie des taquets, le système d'assemblage, entre autres) constituent une composante importante de l'histoire de chaque navire, qui permet de dépasser l'analyse purement technique de l'embarcation et de l'intégrer dans un espace-temps concret, en tant qu'élément social et économique particulier dans un monde de plus en plus global.

Dénommées spécificités régionales, ces variations, dans le cadre de la tradition ibéro-atlantique, traduisent différentes pratiques de chantier qui incorporent des méthodes de construction et des solutions techniques. En dépit de la fragilité de l'information disponible (les variations s'expriment uniquement dans les vestiges archéologiques et le chercheur dispose d'une réalité parcellaire par nature), les indices archéologiques recueillis ont permis d'identifier trois typologies de spécificités :

Techniques et technologiques, traduisant des solutions de conception et de construction spécifiques qui reflètent des développements techniques localisés et/ou le maintien de pratiques ancestrales (Innovation versus Tradition);

Économiques, géographiques et liées à l'accès aux matières premières et aux produits annexes, reflétant différentes capacités d'accès, dues à des facteurs géographiques et économiques ainsi qu'à des réseaux de relations ;

Géographiques associées aux zones de navigation, traduisant des adaptations de la construction à la navigation dans des conditions particulières.

\section{Spécificités techniques et technologiques}

La tradition ibéro-atlantique détenait une situation géographique unique, à la croisée de la Méditerranée classique et de l'Europe du Nord. Il n'y a rien d'étonnant donc à ce que chaque navire se caractérise par des solutions de conception et de construction spécifiques, reflétant des développements techniques localisés et/ou le maintien de pratiques ancestrales. L'analyse des vestiges archéologiques révèle l'influence des espaces maritimes entourant la péninsule Ibérique sur la construction navale, et les réminiscences de traditions ancestrales, comme la construction «bordé premier», sont très claires.

Le renforcement de l'espacement entre les couples ou l'absence de fixation des varangues centrales à la quille traduisent une importance attribuée à la coque 
supérieure à celle qu'elle est supposée détenir théoriquement dans la construction «membrure première», ce qui constitue une résurgence des traditions méditerranéennes. La fixation des galbords à la quille atteste leur mise en place avant le montage de la charpente transversale, un reflet de la tradition de construction «bordé premier». L'intégration des galbords à la quille, est également une réminiscence d'une tradition primitive de construction «bordé premier».

Quant aux différences dans la méthode de recalement des premiers genoux, elles reflètent des innovations en matière de conception développées dans des espaces maritimes extérieurs à la péninsule Ibérique et ont probablement été introduites dans des chantiers ibériques situés dans leurs zones d'influence. D’origine encore inconnue, la légère inclinaison des couples de proue et de poupe vers l'avant et vers l'arrière, respectivement, constitue elle aussi une importante innovation du processus de conception qui a pour effet d'augmenter le fort du navire et sa capacité de charge.

Les spécificités régionales ont toutefois une plus grande importance au niveau des pratiques de chantier, car elles constituent les options du constructeur, bien qu'elles soient imprégnées d'influences intangibles de différentes origines, capables de différencier les embarcations produites dans un certain chantier de leurs homologues.

La fixation d'un seul premier genou à la maîtresse varangue ainsi que le clouage des galbords à la quille attestent différentes séquences de construction. L'absence de talon représente le maintien de préceptes de construction antérieurs à l'introduction de cet élément structurel, pendant que l'absence de râblure dans la région médiane de la quille résulte probablement d'une tentative de simplification du processus de construction. Les taquets taillés dans les varangues, le vaigrage positionné perpendiculairement à la quille ou dépourvu de fixation à la charpente constituent également des spécificités liées directement au chantier d'origine des navires.
Spécificités économiques, géographiques et associées à l'accès aux matières premières et aux produits manufacturés

Comprendre l'existence de facteurs géographiques et économiques associés à l'accès aux ressources matérielles indispensables à la construction navale est éminemment intuitif. L'impact, sur les espèces arborées utilisées dans le navire, de la localisation du chantier par rapport aux zones forestières relève du bon sens. Il n'est donc pas étonnant que le San Diego ait été construit à partir d'essences exotiques orientales. De même, on comprend facilement que la taille d'une quille monoxyle exige que le chantier soit proche de zones forestières encore à demi-vierges ou protégées, dans lesquelles la disponibilité de pièces de bois de grandes dimensions ne constitue pas un problème, ou encore qu'il soit facile d'importer des matières premières.

La contrainte liée à la pénurie de bois approprié à la construction navale a obligé les principales puissances maritimes de l'époque moderne à réserver certaines zones forestières à la construction navale royale $^{23}$ et a probablement astreint les chantiers de plus petites dimensions à utiliser le bois disponible dans la région environnante. La charpente transversale du navire de Ria de Aveiro A est un bon exemple d'utilisation de bois de qualité inférieure à celle requise : des varangues de section et de longueur réduites, très tordues reflètent la taille insuffisante des morceaux de bois en vue de leur transformation en pièces nautiques.

Les ressources financières conditionnaient, quant à elles, l'acquisition de matières premières et/ou de produits annexes, comme la braie ou les tissus. Le volume de capitaux requis par la construction navale étant élevé, il n'est pas improbable que les constructeurs moins pourvus économiquement aient eu recours à l'assemblage en bois au détriment du fer, qu'ils aient mis en œuvre des systèmes de fixation qui n'exigeaient pas d'utiliser du fer et qu'ils aient construis avec les espèces d'arbres à leur disposition, en minimisant les coûts d'importation et de transport de matières premières. 
Tableau 1 :

Indices archéologiques des spécificités identifiées sur les épaves ibéroatlantiques.

\begin{tabular}{|c|c|c|c|}
\hline & Indices archèologiques & RAVA & MA \\
\hline \multirow{18}{*}{ 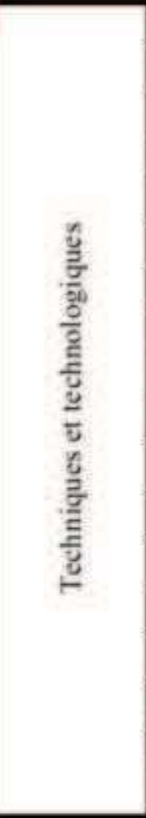 } & \multicolumn{3}{|l|}{ 1. Variations dans le principe de construction } \\
\hline & \multicolumn{3}{|l|}{ Absence de fixation des varangues centrales à la quille } \\
\hline & \multicolumn{3}{|l|}{ Fixation de la totalité des varangues à la quille } \\
\hline & Espacement entre les couples & $\checkmark$ & \\
\hline & \multicolumn{3}{|l|}{ Méthode de basculement/recalement des genoux } \\
\hline & Inclinaison des couples d'avant et d'arrière & & $\checkmark$ \\
\hline & \multicolumn{3}{|l|}{ 2. Diversité des pratiques de chantier } \\
\hline & Fixation d"un seul genou à la maitresse varangue & $\checkmark$ & \\
\hline & \multicolumn{3}{|l|}{ Intégration des galbords dans la quille } \\
\hline & \multicolumn{3}{|l|}{ Clouage des galbords à la quille } \\
\hline & \multicolumn{3}{|l|}{ Absence de râblure dans la région médiane du navire } \\
\hline & \multicolumn{3}{|l|}{ Râblure pas taillèe dans la quille } \\
\hline & \multicolumn{3}{|l|}{ Intégration des taquets aux varangues } \\
\hline & \multicolumn{3}{|l|}{ Individualisation de Templanture du mát } \\
\hline & \multicolumn{3}{|l|}{ Absence de talon } \\
\hline & \multicolumn{3}{|l|}{ Absence daccotars } \\
\hline & \multicolumn{3}{|l|}{ Absence de fixation du vaigrage à la charpente } \\
\hline & \multicolumn{3}{|l|}{ Montage du vaigrage perpendiculairement à la quille } \\
\hline \multirow{7}{*}{ 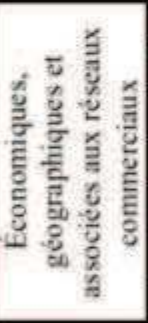 } & Espèces d'arbres utilisées & & \\
\hline & Quille monoxyle & & \\
\hline & Intégration des galbords dans la quille & & \\
\hline & Utilisation de bois de qualité inférieure & $\checkmark$ & \\
\hline & Prédominance ou exclusivité de l'assemblage en fer & & \\
\hline & Prédominance ou exclusivité de l'assemblage en bois & & \\
\hline & Recours à des solutions d'assemblage adaptées an chevillage en bois & & \\
\hline \multirow{8}{*}{ 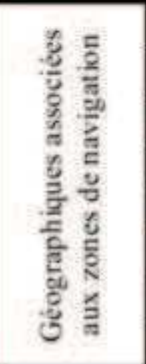 } & Absence d'assemblage entre les tronçons de la quille/talon & & \\
\hline & Espacement entre les couples & $\checkmark$ & \\
\hline & Prédominance ou exclusivité de Tassemblage en fer & & \\
\hline & Prèdominance ou exclusivité de l'assemblage en bois & & \\
\hline & Porques & & \\
\hline & Varangues centrales marquées par un fond plat assez long & & \\
\hline & Fixation de la totalité des varangues à la quille & & \\
\hline & Individualisation de remplanture du màt & & \\
\hline
\end{tabular}

$\begin{array}{lllllll}\text { RAVA } & \text { Ria de Aveiro A } & \text { AGD } & \text { Anga D } & \text { WL } & \text { Western Ledg } & \text { SD } \\ \text { MA } & \text { Marinières } & \text { STB } & \text { Studland Bay } & \text { SJ } & \text { San Juan } & \text { B\&W } \\ \text { CDS } & \text { Cais do Sodre } & \text { HC } & \text { Highborn Cay } & \text { EP } & \text { Emanuel Point } & \text { SW }\end{array}$




\begin{tabular}{|c|c|c|c|c|c|c|c|c|c|c|c|c|}
\hline CDS & AGD & STB & НС & wL & SJ & EP & SD & $B \& W$ & sw & SH I & NSM & AI \\
\hline & & & & & & & & & $\sqrt{ }$ & & & \\
\hline & & & $\checkmark$ & & & & & & & & & \\
\hline & & & & & & & $\checkmark$ & & & & & \\
\hline & & & ( & & & & & & & & & \\
\hline & & & & & & $\sqrt{2}$ & & & & & & $\checkmark$ \\
\hline & & & & & 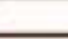 & & & & & & & \\
\hline & & & $\checkmark$ & & & & & $\checkmark$ & & & & \\
\hline & & & & & $\checkmark$ & & & & & & & \\
\hline & & & & $\checkmark$ & & & & & & $\checkmark$ & & \\
\hline & & $\checkmark$ & & & $\checkmark$ & & & & & & & $\checkmark$ \\
\hline & & & & & & & & & & & & \\
\hline & & & & & & & & & & & & \\
\hline$\sqrt{2}$ & & & & & & & & & $\checkmark$ & & & \\
\hline & & $\checkmark$ & & & $\sqrt{2}$ & & 2 & $\frac{2}{2}$ & & & & \\
\hline & & & $\checkmark$ & & & & & & & & & \\
\hline & & & & & & $\checkmark$ & . & & & & & \\
\hline & & & & & & & & & & $\checkmark$ & & \\
\hline & & & & & & & $\sqrt{ }$ & & & & & \\
\hline & & & & & $\checkmark$ & & $\checkmark$ & & & & & \\
\hline & & & & & $\sqrt{2}$ & & & & & & & \\
\hline & & & & & & & & & & & & \\
\hline & & & & & & & & & & & $\checkmark$ & \\
\hline & & & & & & & & & $\checkmark$ & & & $\checkmark$ \\
\hline & & & $\checkmark$ & & & & & & & & & $\checkmark$ \\
\hline$\checkmark$ & & & & & & & & & & & & \\
\hline & & & & & & & $\sqrt{ }$ & & & & & \\
\hline & & & & & & & & & & & $\checkmark$ & \\
\hline & & & & & & & & & $\checkmark$ & & & $\checkmark$ \\
\hline & $\sqrt{ }$ & & & & & & & & & & & \\
\hline & & & & & & & & & & & & $\checkmark$ \\
\hline & & & $\checkmark$ & & & & & & & & & \\
\hline$\checkmark$ & & & & & & & & & $\sqrt{2}$ & & & \\
\hline
\end{tabular}

San Diege B\&W 7

Stonewall
SH 1 Saint Henorat 1

NSM Nossa Senhora Mártires

A) Arade 1 
Toutefois, en ce qui concerne l'accès aux matières premières et aux produits finis, la participation du chantier ou du constructeur aux réseaux commerciaux associés à la fabrication navale était aussi importante que les variables géographiques et économiques. En fait, l'armateur ou le constructeur de petite ou moyenne dimension, même s'il possédait les ressources financières pour payer le prix requis, se trouvait facilement exclu des réseaux commerciaux ${ }^{24}$.

\section{Spécificités géographiques associées aux zones de navigation}

Les méthodes de construction avaient tendance à être adaptées à la zone de navigation, au(x) parcours prédéfini(s) pour le navire. En effet, selon qu'elles étaient destinées à naviguer dans des eaux abritées ou intérieures, près de la côte ou en haute mer, les embarcations étaient exposées à des efforts structurels et à des contraintes de nature différente.

Si la navigation dans des eaux abritées, comme des fleuves, des lagunes, l'intérieur de zones portuaires ou des régions lacustres, était partiellement protégée des vagues, des vents, des courants et des marées, elle était exposée, par contre, aux constantes modifications du fond et des chenaux de navigation, en raison de la décharge de lests, des ensablements et désensablements abrupts, de la construction d'infrastructures. Ces embarcations avaient naturellement tendance à présenter des fonds plats ou légèrement bombés et un faible tirant d'eau.

La navigation côtière, entre des zones portuaires, quant à elle, exigeait déjà des navires à la coque et aux apparaux aptes à la navigation à la bouline et de dimensions, de tirant d'eau et de stabilité supérieurs, capables d'affronter des conditions maritimes qui n'étaient pas toujours favorables mais, dans le même temps, adaptés à l'entrée dans les estuaires. Un fond plat avec des varangues assez longues dans la région centrale, par exemple, permettaient aux navires de naviguer avec la stabilité requise aussi bien en mer profonde que sur les fleuves de faible profondeur.
Pour la navigation océanique, entre des ports nationaux et étrangers, hors des limites de visibilité de la côte, les embarcations étaient conçues comme de véritables cités flottantes. En mer des mois durant, luttant contre des phénomènes météorologiques extrêmes, les navires étaient dotés de structures renforcées, comme des coques et des quilles doubles et des porques, des pièces proéminentes et dûment assemblées entre elles, ainsi que plusieurs mâts puissants ou un système d'assemblage adapté ${ }^{25}$. (Tableau 1 : Indices archéologiques des spécificités identifiées sur les épaves ibero-atlantiques)

\section{Conclusion}

Aujourd'hui encore dans le cadre de la construction navale traditionnelle, ce n'est que des mains d'un même constructeur que peuvent sortir deux navires identiques. Pendant le haut Moyen-Âge et à l'époque moderne, chaque constructeur avait ses procédés empiriques et il serait illusoire de croire qu'une certaine règle d'un maître charpentier du $\mathrm{XV}^{\mathrm{e}}$ siècle puisse devenir universelle et être suivie en tous points par un autre. Les spécificités décrites attestent donc la diversité des solutions de construction et ne représentent guère plus qu'une première approche d'une thématique complexe et difficile à saisir.

Du point de vue technique et technologique, les variations dans le principe de construction témoignent de spécificités de fond liées au système architectural ibéroatlantique proprement dit, révélant des influences d'espaces maritimes extra-péninsulaires et probablement des réminiscences de traditions de construction ancestrales. La diversité des pratiques de chantier, ellemême, doit être entendue comme un contexte plus vaste dans lequel l'influence d'autres espaces maritimes joue certainement un rôle très important, tout comme la tradition de construction transmise en secret de maître à apprenti. Toutefois, les différences dans la séquence de construction, dans les apparaux des embarcations, dans la typologie des écarts et des assemblages, entre autres, sont également liées à la propre capacité d'évolution des différents chantiers, à la 
rotation et la spécialisation de la main-d'œuvre et aux contacts avec des navires provenant de différents ports nationaux et étrangers.

La typologie et la géographie du chantier revêtent également de l'importance dans le cadre de l'accès aux matières premières et aux produits annexes. Les espèces d'arbres ainsi que la dimension et la qualité des pièces de bois traduisent la proximité de zones forestières spécifiques. À son tour, la prédominance ou l'utilisation exclusive du chevillage/assemblage en bois ou en fer reflète la typologie, la dimension et la capacité financière du chantier - des caractéristiques qui, associées à une région d'origine, peuvent contribuer à l'insertion de l'arsenal/du chantier dans les réseaux commerciaux et d'influence de l'époque.

Les spécificités techniques, technologiques et de ressources nous permettent, en outre, de lever le voile sur un autre type d'information concernant les navires ibéro-atlantiques, par exemple, la répartition du travail sur le chantier et, par conséquent, la dimension du chantier ou l'essor de la construction dans la région ${ }^{26}$.

La corrélation des spécificités observées dans les différents ibéro-atlantiques avec des espaces géographiques concrets et l'identification de sous-groupes de navires, distincts du point de vue de leur paysage culturel maritime, à son tour, n'est pas impossible, mais elle requiert une solide base d'informations. Le développement d'une base de données relative aux spécificités ibéro-atlantiques permettra à long terme de comprendre plus précisément la genèse de cette tradition de construction et ses influences respectives. Pour l'instant, une chose est certaine, la tradition de construction ibéro-atlantique ne peut déjà plus être envisagée isolément : "perhaps the concept of a continuum of several interrelated "traditions" is better suited to describe the reality of [this] ancient hull structure ${ }^{27}$.

1 McGRAIL Seán, « Romano-Celtic boats and ships : characteristic features », dans International Journal of Nautical and Archaeology, 24.2, 1995, p. 140.
2 RIETH Éric, « Construction navale à franc-bord en Méditerranée et Atlantique (XIV ${ }^{\mathrm{e}}-\mathrm{XVII}{ }^{\mathrm{e}}$ siècles) et " Signatures architecturales » : une première approche archéologique », dans Méditerranée antique. Pêche navigation, commerce, Paris, CTHS, 1998, p. $177-188$.

3 OERTLING Thomas, «The few remaining clues...», dans ARNOLD III J. Béat, Underwater Archaeology Proceedings from the Society for Historical Archaeology Conference, Pleasant Hill, Society for Historical Archaeology, 1998, p. 102

4 ALVES Francisco (ed.), Proceedings. International Symposium on Archaeology of Medieval and Modern Ships of Iberian-AtlanticTradition. Hull remains, manuscripts and ethnographic sources : a comparative approach, Lisbon, Centro Nacional de Arqueologia Náutica e Subaquática / Academia de Marinha, 2001.

5 ALVES Francisco, RODRIGUES Paulo et al., « Aproximação arqueológica às fontes escritas da arquitectura naval portuguesa », dans GUERREIRO Inácio, DOMINGUES Francisco C., Fernando Oliveira e o Seu Tempo. Humanismo e Arte de Navegar no Renascimento Europeu (1450-1650). Actas da IX Reunião Internacional da História da Náutica e da Hidrografia, Cascais, Éd. Patrimonia, 2000, p. 225.

6 OERTLING Thomas, « The concept of the Atlantic Vessel », dans ALVES Francisco, 2001, op cit, p. 234.

7 OERTLING, 2001, op. cit.

8 OERTLING, 2001, op. cit., p. 233-240.

9 L'effort monographique qui a eu lieu au cours de la première décennie du XXI ${ }^{\text {e }}$ siècle ainsi que l'analyse des dernières épaves ibéro-atlantiques identifiées échappent à cette perspective théorico-comparative. À noter que la dernière matrice publiée ne tenait compte que des épaves de Rye A (Sussex/ Royaume-Uni, $\mathrm{XVI}^{\mathrm{e}}$ siècle), San Juan (Labrador/ Canada, XVI ${ }^{\mathrm{e}}$ siècle), San Esteban (île Padre/ États-Unis, XVI ${ }^{\mathrm{e}}$ siècle), Highborn Cay (Bahamas, XVI ${ }^{\mathrm{e}}$ siècle), Molasses Reef (îles Turks and Caicos/ Royaume-Uni, XVI ${ }^{\mathrm{e}}$ siècle), Cattewater (Plymouth/ RoyaumeUni, XVI ${ }^{\mathrm{e}}$ siècle), Studland Bay (Dorset/ Royaume-Uni, XVI ${ }^{\mathrm{e}}$ siècle), Emanuel Point (Floride/ États-Unis, XVI ${ }^{\mathrm{e}}$ siècle), Ria de Aveiro A (Aveiro/ Portugal, XV ${ }^{\mathrm{e}}$ siècle), Western Ledge (Bermudes, XVI ${ }^{\mathrm{e}}$ siècle), St. John's Bahamas (Bahamas, XVIe siècle), Angra D (Açores/ Portugal, XV / XVI ${ }^{\mathrm{e}}$ siècles), Nossa Senhora dos Mártires (Lisbonne/ Portugal, XVII ${ }^{\mathrm{e}}$ siècle), Cais do Sodré (Lisbonne/ Portugal, $\mathrm{XV}^{\mathrm{e}} / \mathrm{XVI}^{\mathrm{e}}$ siècles) et Corpo Santo (Lisbonne/ Portugal, XIV ${ }^{\mathrm{e}}$ siècle).

10 Aux 15 épaves considérées dans la dernière matrice publiée sur la tradition ibéro-atlantique, ont été ajoutées les épaves de Cape Carbonara (Sardaigne/ Italie, $\mathrm{XV}^{\mathrm{e}}$ siècle), Marinières (Villefranche-sur-Mer/ France, $\mathrm{XV}^{\mathrm{e}}$ siècle), Boudeuse Cay (Seychelles, $\mathrm{XVI}^{\mathrm{e}}$ siècle), San Diego (Manille/ Philippines, XVI ${ }^{\mathrm{e}}$ siècle), B\&W 7 (Copenhage/ Danemark, XVI ${ }^{\mathrm{e}}$ siècle), Arade 1 (Portimão/ Portugal, XVI ${ }^{\mathrm{e}}$ siècle), Stonewall (Bermudes/ Royaume-Uni, XVII ${ }^{\mathrm{e}}$ siècle) et Saint-Honorat 1 (Cannes/ France, XVII ${ }^{\mathrm{e}}$ siècle). 
11 La croyance en l'existence d'un modèle mental abstrait, sous-jacent à la construction navale, n'est pas un apanage de la Renaissance. Basch soutient qu'il en existait déjà dans les chantiers de l'Antiquité égyptienne. Toutefois, c'est à Crumlin-Pederson que l'on doit d'avoir reconnu, sur le plan archéologique, l'existence d'un modèle mental prédéterminé dans l'analyse des navires de Skuldelev 1 et 3, datés du XI ${ }^{\mathrm{e}}$ siècle. BASCH Lucien, « Recherche d'une généalogie », dans Archaeologia Maritima Mediterranea, 5, 2008, p. 69-81; CRUMLIM-PEDERSON Ole, OLSEN Olaf, The Skuldelev Ships I, Roskilde, The Viking Ship Museum/ Center for Maritime Archaeology of the National Museum of Denmark, 2002, p. 234.

12 L'utilisation de ce terme se rapporte à la mécanisation du processus de production, visant à réduire le temps nécessaire à l'armement d'un navire, le risque naturellement associé à la capacité hydrodynamique finale et la périodicité de sa maintenance ainsi qu'à maximaliser le cycle de vie des embarcations.

13 GARCIA Catarina, MONTEIRO Paulo, " The excavation and dismantling of Angra D, a probable Iberian seagoing ship, Angra Bay, Terceira Island, Azores, Portugal. Preliminary assessment », dans ALVES Francisco, 2001, op. cit., p. 437.

14 LOEWEN Brad, " La carène : de la conception et de la construction », dans GRENIER Robert et al., L'archéologie subaquatique de Red Bay. La construction navale et la pêche de la baleine basques au XVI siècle, Canada, Parcs Canada, 2007, III, p. 57.

15 LOUREIRO Vanessa, L'Épave Arade 1 : l'influence des chantiers régionaux sur la tradition de construction navale ibéro-atlantique, Mémoire de Doctorat, Paris, Université de Paris 1 - Panthèon Sorbonne, 2011, p. 159-172.

16 SMITH R. C., BRATTEN J. R., COZZI J., PLASKETT K., The Emanuel Point Ship. Archaeological Investigations : 1997-1998, Florida, Archaeology Institute of West Florida, 1998.

17 CARRÉ D., DESROCHES J.-P., GODDIO F., Le San Diego. Un trésor sous la mer, Paris, Association française d'action artistique/ Ministère des aff aires étrangères/ Réunion des musées nationaux, 1994

18 CASTRO Filipe, «The Remains of a Portuguese Indiaman at Tagus Mouth, Lisbon, Portugal (Nossa Senhora dos Mártires, 1606?)», dans ALVES Francisco, 2001, op. cit., p. 387-390.

19 LAVANHA João Batista, Livro Primeiro da Architectura Naval, Lisboa, Academia da Marinha, 1608-1616 ; OLIVEIRA Fernando, O Liuro da Fábrica das Naus, Lisboa, Academia da Marinha, 1580 .

20 Sur le plan archéologique, ce système de conception peut être attesté par le biais de l'identification de marques incisées (traits d'astilhas, traits de l'escoue et de la limite du plat des varangues), comme dans les épaves de Ria de Aveiro A, Cais do Sodré, San Juan, Arade 1 ou Nossa Senhora dos Mártires.

21 LOUREIRO Vanessa, 2011, op cit., p. 253-259.

22 LOEWEN Brad, 2007, op. cit., p. 82-83.
23 AZEVEDO Orlando Vasconzelos, A floresta e o domínio do mar, Lisboa, Academia de Marinha, 1997, p. 26 ; COSTA Leonor Freire, Naus e galeões na Ribeira de Lisboa. A construção naval no século XVI para a rota do Cabo, Cascais, Patrimonia, 1997, p. 308-324.

24 BARROS Amândio Jorge Morais, « O grande comércio dos "pequenos actores". Portuenses e Bascos na construção do sistema atlântico », dans Revista Internacional de los Estudios Vascos, 50, 2, 2005, p. 349-350.

25 L'idée que la typologie du système d'assemblage constitue une caractéristique régionale au sein de la construction navale, date du XVII ${ }^{e}$ siècle. Lavanha souligne qu'alors que les navires de l'Europe du Nord pouvaient recourir au chevillage en bois, les embarcations qui naviguaient dans des eaux chaudes devaient être clouées en fer afin d'éviter le taredo navalis. Plus récemment, plusieurs auteurs ont confirmé la corrélation entre les systèmes de fixation des embarcations et des zones possibles de navigation. LAVANHA, op. cit., 1608-1616, p. 33 ; ADAMS Jon, VAN HOLK André F. L., Maarleveld Thijs J., Dredgers and Archaeology. Ship Finds from the Slufter, Netherlands, WVC, 1990, p. 83-91; LOEWEN Brad, « Le vaigrage, les ponts et les châteaux : une charpenterie adaptée pour la pêche de la baleine ", dans GRENIER Robert et al, 2007, op. cit, p. 256.

26 Dans l'épave San Juan, le clouage en fer a été utilisé uniquement pour la fixation provisoire des planches et des varangues, en procédant par la suite à leur chevillage en bois : «the use of iron nails allowed the carpenters to assemble the hull rapidly and independently of the treenailers, who arrived at the shipyard at different moments and were able to work on more than a ship at once ». LOEWEN, 2007, op. cit.

27 THOMSEN Mikkel, « The Studland Bay wreck, Dorset, UK : hull analysis », dans The International Journal of Nautical Archaeology, 29.1, 2000, p. 83. 